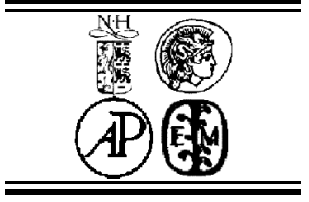

\title{
Atomic structure of threading dislocations in AlN thin films
}

\author{
Yuki Tokumoto, ${ }^{\mathrm{a},}{ }^{*}$ Naoya Shibata, ${ }^{\mathrm{b}, \mathrm{c}}$ Teruyasu Mizoguhci, ${ }^{\mathrm{b}}$ Takahisa Yamamoto, ${ }^{\mathrm{d}}$ \\ Yuichi Ikuhara ${ }^{\text {be }}$ \\ ${ }^{a}$ Institute for Materials Research, Tohoku University, 2-1-2, Katahira, Aoba-ku, Sendai 980-8577, Japan \\ ${ }^{b}$ Institute of Engineering Innovation, School of Engineering, The University of Tokyo, 2-11-16, Yayoi, Bunkyo, Tokyo 113-8656, Japan \\ ${ }^{c}$ PRESTO, JST, 4-1-8, Honcho, Kawaguchi, Saitama 332-0012, Japan \\ ${ }^{d}$ Department of Advanced Materials Science, Graduate School of Frontier Science, The University of Tokyo, 5-1-5, Kashiwanoha, Kashiwa, \\ Chiba 277-8651, Japan
}

${ }^{e}$ WPI Advanced Institute for Materials Research, Tohoku University, 2-1-2, Katahira, Aoba-ku, Sendai 980-8577, Japan

Elsevier use only: Received date here; revised date here; accepted date here

\begin{abstract}
The core structure of threading dislocations in AlN films was investigated by atomic-resolution scanning transmission electron microscopy. The threading dislocations in the AlN films were found to be mostly edge-type perfect dislocations. It was directly revealed that the edge dislocation core has the 8-atom ring structure which is an energetically favorable structure predicted by previous theoretical studies. (c) 2001 Elsevier Science. All rights reserved
\end{abstract}

Key words: AlN; dislocations; HAADF STEM

\section{Introduction}

Group III nitrides have attracted much attention because of their potential applications for short-wavelength laser devices [1,2] and high-power electronic devices [3]. In the epitaxial growth of group III nitride films on sapphire $(\alpha-$ $\mathrm{Al}_{2} \mathrm{O}_{3}$ ) substrates, it has been known that the mosaic growth occurs due to the large lattice mismatch between the film and the substrate. In this case, films are formed by sub-grains slightly misoriented relative to each other around the $c$ axis $[4,5]$. As a result, high-density threading dislocations are introduced at the sub-grain boundaries in order to compensate slight crystal misorientations [4-6].

Device properties of materials with such high-density threading dislocations are strongly influenced by the properties of individual dislocations [7-9]. Therefore, it is important to clarify the properties of each individual dislocation in order to understand the device properties of the whole materials. The optical or electrical properties of dislocations are considered to depend on their atomic-scale core structures $[10,11]$. However, it is still difficult to clearly determine the atomic-scale detailed structures of dislocation cores. So far, concerning the edge-type threading dislocations in wurtzite group III nitride films, two types of core structures, namely the 8-atom and 5/7atom ring structures, have been reported $[12,13]$. In $\mathrm{GaN}$ films grown by metal-organic vapor phase epitaxy (MOVPE), the 8-atom ring structure has been observed using atomic-resolution high-angle annular dark field (HAADF) scanning transmission electron microscopy (STEM) [12]. In GaN films grown by molecular beam epitaxy (MBE), both the 8-atom and 5/7-atom ring structures have been observed using high-resolution transmission electron microscopy (HRTEM) [13]. Concerning the edge-type threading dislocations in AlN thin films, although the Burgers vector and the formation

${ }^{*}$ Corresponding author. Tel.: +81-22-215-2044; fax: +81-22-215-2041; e-mail: y.tokumoto@imr.tohoku.ac.jp

(C) 2009. This manuscript version is made available under the Elsevier user license http://www.elsevier.com/open-access/userlicense/1.0/ 
mechanism have been investigated [4,5], the atomic-scale dislocation core structure has not been revealed. In this study, we directly observed atomic-scale core structure of threading dislocations in AlN films by using atomicresolution HAADF STEM [14].

\section{Experiments}

Commercially available AlN films grown on (0001) sapphire substrates by MOVPE (DOWA Electronics Materials Co., Ltd.) were used in this study. Plan-view and cross-sectional specimens for TEM observations were prepared by conventional methods including mechanical polishing, dimple grinding and Ar-ion-beam thinning processes. Conventional TEM observations were carried out using a 200-kV TEM (JEM-2010HC, JEOL Ltd). Atomic-resolution images were taken with a $200-\mathrm{kV}$ STEM (JEM-2100F, JEOL Ltd.) equipped with a spherical aberration $\left(\mathrm{C}_{\mathrm{s}}\right)$-corrector (CEOS Co.), providing a minimum electron probe diameter of about $0.1 \mathrm{~nm}$.

\section{Results and discussion}

Plan-view (0001) bright-field TEM image and the corresponding selected area diffraction pattern of the film are shown in Figs. 1(a) and (b), respectively. Dark dot-like contrasts in Fig. 1(a) correspond to the threading dislocations formed parallel to the film growth direction. The density of the threading dislocations was estimated to be about $4 \times 10^{10} \mathrm{~cm}^{-2}$. From Fig. 1(b), it was confirmed that the film is single-crystalline wurtzite AlN. Based on the diffraction pattern obtained from the interface between the AlN film and the sapphire substrate, the orientation relationship for the interface was confirmed to be (0001)AlN//(0001)sapphire

and

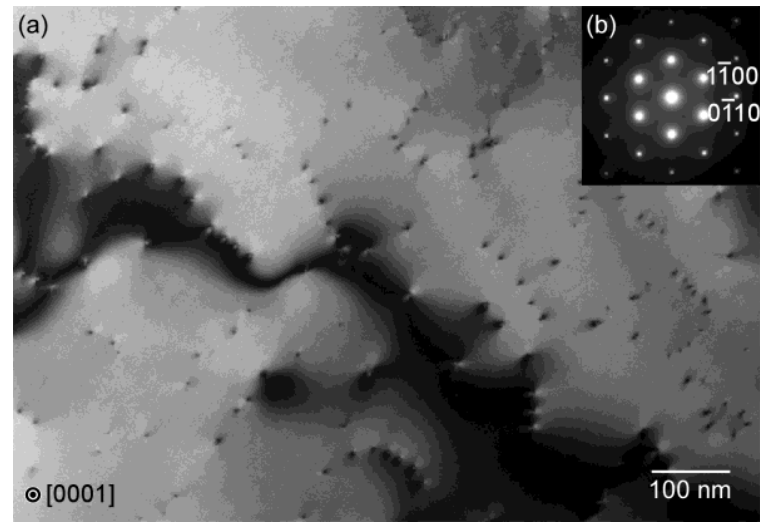

Fig. 1. (a) Plan-view (0001) bright-field TEM image of the film. Dark dot-like contrasts correspond to threading dislocations. (b) Selected area diffraction pattern of the film. It was shown that the film is single-crystalline wurtzite AlN.
$[1 \overline{1} 00] \mathrm{AlN} / /[11 \overline{2} 0]$ sapphire.

In wurtzite structure, the threading dislocations along the <0001> direction can have three types of characters: edge, screw or mixed. In order to clarify the character of the threading dislocations, cross-sectional TEM observations were performed. Figs. 2(a) and (b) show cross-sectional dark-field TEM images of the AlN film. Fig. 2(a) was taken in two-beam diffracting condition with $\mathrm{g}=11 \overline{2} 0$, where the contrast of pure screw-type threading dislocations becomes extinct, while that of pure edge- or mixed-type threading dislocations is imaged. On the other hand, Fig. 2(b) was taken in two-beam diffracting condition with $\mathrm{g}=000 \overline{4}$, where the contrast of pure edge-type threading dislocations becomes extinct, while that of pure screw- or mixed-type threading dislocations is imaged. The TEM images in Figs. 2(a) and (b) were obtained from the same area. Based on the dark-field TEM images, the ratio of edge and screw-type dislocations was estimated to be about 1:10. Therefore, the threading dislocations in the AIN films are considered to be mostly edge-type ones. In Fig. 2(b), bright contrasts were also observed in the vicinity of the interface between the AlN film and the sapphire substrate, which suggests the presence of half-loops with screw component. Similar defects have been observed in GaN films grown on $6 \mathrm{H}-\mathrm{SiC}$ (0001) substrates [15]. These defects may be formed as a result of film growth processes with sub-grains slightly tilted with respect to the substrate.

Fig. 3(a) shows a HAADF-STEM image of an edge dislocation in the AlN film viewed along the <0001> direction. Bright spots directly correspond to the position of atomic columns of alternating $\mathrm{Al}$ and $\mathrm{N}$ atoms. It was found that the edge dislocations formed in the films are perfect dislocations with the Burgers vectors of $\mathbf{b}=1 / 3<1 \overline{2} 10\rangle$ type. Fig. 3 (b) shows a schematic of the core structure observed in the HAADF-STEM image shown in Fig. 3(a). Al and $\mathrm{N}$ atoms are denoted by white

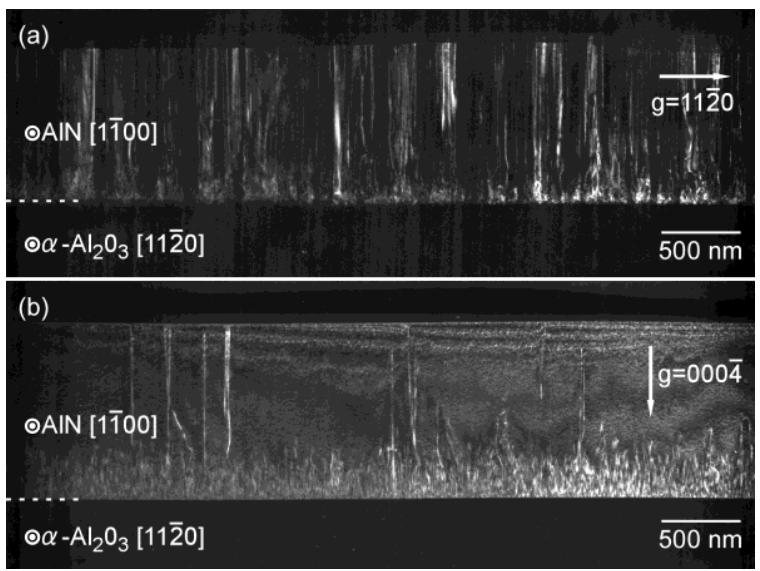

Fig. 2. Cross-sectional dark-field TEM images of the AlN film on the sapphire substrate, which are taken in two-beam diffracting condition with (a)g=112 0 and (b)g $=000 \overline{4}$, respectively. 


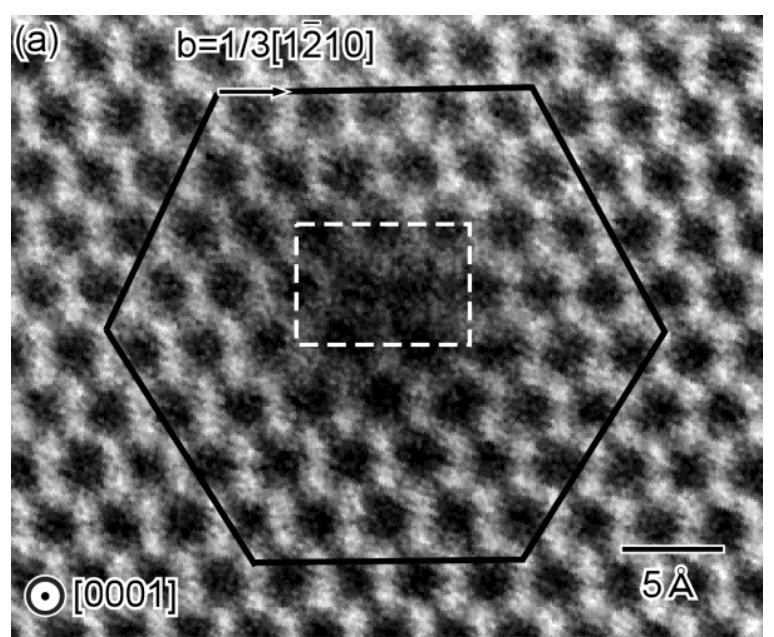

(b)

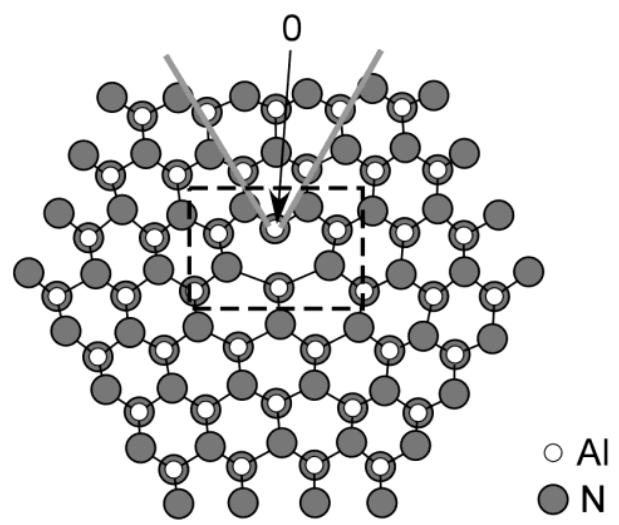

Fig. 3. (a) HAADF-STEM image of a dislocation core of the AlN film viewed along the <0001> direction. Bright spots correspond to the position of atomic columns of alternating $\mathrm{Al}$ and $\mathrm{N}$ atoms. It was indicated that the dislocation is a perfect edge dislocation with the Burgers vector of $\mathbf{b}=1 / 3<1 \overline{2} 10>$. (b) Schematic of the core structure observed in the HAADFSTEM image shown in (a). Al and $\mathrm{N}$ atoms are denoted by white and gray circles, respectively. The dislocation core is surrounded by dashed lines in (a) and (b). The two extra halfplanes are indicated by gray lines, and the atomic column where the two extra half-planes are terminated is labeled ' 0 ' in (b). It was indicated that the edge dislocation has the 8-atom ring core structure.

and gray circles, respectively. The dislocation core is surrounded by dashed lines in Figs. 3(a) and (b). In Fig. 3(b), two extra half-planes are indicated by gray lines, and the atomic column where the two extra half-planes are terminated is labeled ' 0 '. It is found that the dislocation core is surrounded by 8 atomic columns, which indicates that the edge dislocations in the AlN film should have the 8 -atom ring core structure. As mentioned above, two types of core structures, the 8-atom and 5/7-atom ring structures, have been reported for edge dislocations with $\mathbf{b}=1 / 3<1 \overline{2} 10\rangle$ in wruzite structre $[12,13]$. The 5/7-atom ring structure can be formed by removing the atomic column labeled ' 0 ' at the core of the 8-atom ring structure. According to the theoretical study of dislocation core structures in $\mathrm{AlN}$, it is reported that the 8-atom ring structure is more energetically favorable than the 5/7-atom ring structure [16]. We have observed more than 10 edge dislocations in the AIN film, and all the dislocations were found to have the 8-atom ring core structures. Thus, the 8atom ring structure should be the stable structure for the dislocation core in AlN, which is consistent with the previous theoretical study. The possibility of the presence of $\mathrm{N}$ vacancies or $\mathrm{Al}$ vacancies should be considered as a future work. However, direct confirmation of the formation of the 8-atom ring structure will be important structural basis for understanding the optical or electrical properties of dislocations in AlN.

\section{Conclusions}

Threading dislocations in AlN films grown on sapphire substrates were investigated by TEM and HAADF STEM. It was revealed that the majority of the threading dislocations were perfect edge-type dislocations with the Burgers vector of $\mathbf{b}=1 / 3<1 \overline{2} 10>$. Furthermore, the dislocations were shown to have the 8 -atom ring core structure. The present result is consistent with the previous theoretical study of the stable dislocation core structure in AlN [16].

\section{Acknowledgments}

This work was supported in part by the Grant-in-Aid for Scientific Research on Priority Areas Nano Materials Science for Atomic-scale Modification 474 from the Ministry of Education, Culture, Sports and Technology (MEXT) of Japan. Y.T. was supported as a Japan Society for the Promotion of Science (JSPS) research fellow. Y.T. acknowledges financial support received from the Kazato Research Foundation, Japan. N.S. acknowledges support from PRESTO, Japan Science and Technology Agency.

\section{References}

[1] S. Nakamura, T. Mukai, M. Senoh, J. Appl. Phys. 76 (1994) 8189.

[2] S. Nakamura, M. Senoh, S. Nagahama, N. Iwasa, T. Yamada, T. Matsuhsita, H.Kiyoku, Y. Sugimoto, T. Kozaki, H. Umemoto, M. Sano, K. Chocho, Appl. Phys. Lett. 72 (1998) 211.

[3] Q. Chen, M. A. Khan, J. W. Yang, C. J. Sun, M. S. Shur, 
H. Park, Appl. Phys. Lett. 69 (1996) 794.

[4] Y. Tokumoto, N. Shibata, T. Mizoguchi, M. Sugiyama, Y. Shimogaki, J. -S. Yang, T. Yamamoto, Y. Ikuhara, J. Mater. Res. 23 (2008) 2188.

[5] Y. Tokumoto, N. Shibata, T. Mizoguchi, T. Yamamoto, Y. Ikuhara, Microsc. Microanal. 14 (2008) 258.

[6] X. J. Ning, F.R. Chien, P. Pirouz, J.W. Yang, M. Asif Khan, J. Mater. Res. 11 (1996) 580.

[7] P. J. Hansen, Y. E. Strausser, A. N. Erickson, E. J. Tarsa, P. Kozodoy, E. G. Brazel, J. P. Ibbetson, U. Mishra, V. Narayanamurti, S. P. DenBaars, J. S. Speck, Appl. Phys. Lett. 72 (1998) 2247.

[8] N. G. Weimann, L. F. Eastman, D. Doppalapudi, H. M. Ng, T. D. Moustakas, J. Appl. Phys. 83 (1998) 3656.

[9] H. Hasegawa, Y. Kamimura, K. Edagawa, I. Yonenaga, J. Appl. Phys. 102 (2007) 026103.

[10] J. Elsner, R. Jones, P. K. Sitch, V. D. Porezag, M. Elstner, Th. Frauenheim, M. I. Heggie, S. Öberg, P. R. Briddon, Phys. Rev. Lett. 79 (1997) 3672.

[11] A. F. Wright, U. Grossner, Appl. Phys. Lett. 73 (1998) 2751.

[12] Y. Xin, S. J. Pennycook, N. D. Browning, P. D. Nellist, S. Sivananthan, F. Omnès, B. Beaumont, J. P. Faurie, P. Gibart, Appl. Phys. Lett. 72 (1998) 2680.

[13] V. Potin, P. Ruterana, G. Nouet, R. C. Pond, H. Morkoç, Phys. Rev. B 61 (2000) 5587.

[14] S. J. Pennycook, D. E. Jesson, Phys. Rev. Lett. 64 (1990) 938.

[15] F. R. Chien, X. J. Ning, S. Stemmer, P. Pirouz, M. D. Bremser, R. F. Davis, Appl. Phys. Lett. 68 (1996) 2678.

[16] A. F. Wright, J. Furthmüller, Appl. Phys. Lett. 72 (1998) 3467. 